\title{
1 An approach to estimating flame radiation in combustion chambers 2 containing suspended-particles
}

3 Zhiwei Yang, Adewale Adeosun, Benjamin M. Kumfer, and Richard L. Axelbaum*

4

5 Mailing Address:

6 Department of Energy, Environmental \& Chemical Engineering, Consortium for Clean

7 Coal Utilization, Washington University in St. Louis, St. Louis, Missouri 63130, USA

8

9 * Corresponding author. Tel: +1 314-935-7560; Fax: +1 314-935-5464.

10 Email: axelbaum@wustl.edu (Prof. Richard L. Axelbaum)

11

12

13

14

15

16

17

18

19

20

21 


\section{Abstract}

23 Thermal radiation often plays a key role in heat transfer between flames and the

24 surrounding environment. Therefore, reliable measurement of flame radiation is

25 indispensable in the study of combustion. While the measurement of radiative flux from

26 flames is straightforward in an open environment, it is challenging to do so in a confined

27 chamber, due to the influence of the walls. Radiation emitted by the walls and the

28 reflection of flame radiation off the walls can interfere with measurements of flame

29 radiation. For wall surfaces that are at high temperature or highly reflective, the error in

30 the flame radiation measurement can be significant.

32 In this paper, a theoretical analysis was carried out to study the contribution of wall

33 emission and wall reflection to the incident radiation measured by radiometers. The

34 results reveal that for a typical lab-scale air-fired flame, the contribution of wall emission

35 and wall reflection to the measured incident radiation can be as high as $55 \%$ for a

36 refractory-lined wall and $40 \%$ for a water-cooled wall. A convenient approach is

37 proposed to distinguish flame radiation from the measured incident radiation. The

38 approach is based on measurements of surface incident radiation and net radiative heat

39 flux through the wall. The approach can be applied to optically thin systems (most lab-

40 scale and some pilot-scale atmospheric pressure combustion systems) and optically dense

41 systems (typically pressurized large-scale combustion systems). CFD simulations were

42 also performed for two coal-fired combustors, one which represents optically thin and the

43 other optically thick systems, to validate the theoretical model and the approach to

44 estimating flame radiation. Good agreement was observed between CFD results and the 
45 theoretical model results, and the proposed approach to estimation of flame radiation was

46 found to have reasonable accuracy.

48 Key words: thermal radiation, flame, combustion, wall emission, wall reflection

\section{1. Introduction}

52 Thermal radiation quite often plays an important role in heat transfer between flames and 53 the surrounding environment. In fact, flame radiation caused by gas and particles is the 54 dominant heat transfer mode in many combustion applications, such as industrial 55 furnaces and coal-fired combustion boilers [1-5]. Butler and co-workers performed

56 laboratory-scale experiments to understand heat transfer by thermal radiation in a 57 pulverized coal-fired reactor, and they reported that the thermal radiation from coal

58 flames can be as high as $90 \%$ of the total heat transfer [6, 7]. Moreover, radiation has a 59 significant influence on flame ignition, flame structure, and flame spread [1]. Park and

60 Tien showed that the absorption of incident radiation by the gas phase in the near-burner

61 region is one of the critical mechanisms responsible for gas-phase ignition of solid fuels

62 when exposed to strong external thermal radiation [8]. A reliable measurement of flame

63 radiation is indispensable to the study of combustion. As new technologies are developed

64 to address environmental challenges associated with flames and combustion, the need for

65 flame radiation measurement will become increasingly important, especially for the 66 design and optimization of advanced combustion systems [9, 10].

67 
68 While the measurement of radiation coming from flames is straightforward in an open

69 environment [11], it is challenging to do so in a confined chamber, due to the influence of

70 the walls. Radiation emitted from the walls and flame radiation that is reflected off the

71 walls can interfere with the measurement of flame radiation. The difficulty of

72 distinguishing flame radiation from the measured incident radiation has been emphasized

73 in several studies [12-15]. Also, determination of flame radiation through computational

74 calculations remains a subject with high uncertainty in practical combustion systems [16-

75 19]. This is principally due to the complexity associated with determining the key

76 radiative properties, such as the local absorption and scattering coefficients of optically

77 active gaseous components and, more importantly, those of various particulates (coal,

78 char, ash, and soot) [20-24].

79

80 As shown in Fig. 1, the surface incident radiation received by a radiometer at the inner

81 surface of a combustion chamber has three components:

82 (1) radiation coming from the flame and combustion products (i.e., from the particles and 83 optically active gases),

84 (2) radiation emitted from the walls, and

85 (3) radiation reflected by the walls.

86 In addition, in all three components the received radiation is partly absorbed and scattered

87 by the gas/particle medium on its way to the wall. The first component above is referred 88 to as "flame radiation" in this study.

89

90 
91 The surface incident radiation, $G_{t o t a l}$, measured by a radiometer can be expressed as

$92 G_{\text {total }}=E_{\text {flame }}+G_{\text {wall }}$,

93 where $E_{\text {flame }}$ is the flame radiation (i.e. radiation directly coming from the high

94 temperature combustion products and partly absorbed and scattered by the medium on its

95 way), and $G_{\text {wall }}$ is the combined contribution of wall emission and wall reflection to the

96 surface incident radiation. As will be shown later, in many combustion applications, $G_{\text {wall }}$

97 is comparable to or even higher than $E_{\text {flame}}$, especially when the wall surfaces are at high

98 temperature or are highly reflective. The strong impact of the wall on the radiative

99 measurement can lead to difficulties in fundamental studies of combustion. For example,

100 the difference in flame radiation caused by varying operating parameters, such as the

101 stoichiometric ratio and swirl number, can be buried in the dominating effect of wall

102 emission and/or wall reflection when the wall surfaces are hot and/or reflective.

103

104 In order to distinguish flame radiation from measured incident radiation, some

105 researchers measured incident radiation with and without the flame (i.e., immediately

106 after the flame is shut off) and used the difference between the two to approximate flame

107 radiation [12]. While this approach addresses the influence of wall emission, it does not

108 take into account of the influence of wall reflection, which, in some circumstances, can

109 be significant, as will be shown later.

111 Others tried to block most of the radiation caused by the walls [13, 14, 25, 26] by 112 utilizing a narrow angle radiometer together with a non-reflecting, cold surface 113 positioned on the wall opposite the radiometer. While this approach can minimize the 
114 effects of wall emission and reflection, it provides only line-of-sight information about

115 the radiative characteristics in the chamber. However, in most circumstances,

116 hemispherical radiative heat flux is of more interest because it is directly related to the

117 design and operation of combustors. For example, in the power industry, material

118 selection for water-tube walls and the arrangement of heat exchangers are determined by

119 the magnitude of hemispherical heat flux in the combustion boilers. In addition,

120 hemispherical radiative flux measurements provide information that is not biased by the

121 non-homogeneous distribution of particle number density in the combustion chamber,

122 unlike the narrow angle measurement, which may vary wildly with directions [27]. While

123 a number of studies have reported measurement of hemispherical radiation in various

124 combustion chambers [3, 4, 12, 28, 29], and other studies have tried to design and

125 improve probes for hemispherical radiative measurement [30-32], few studies have

126 discussed how to distinguish flame radiation from the incident radiation that is received

127 by these hemispherical radiative measurement probes.

129 The purpose of this work is to develop a theoretical approach to quantify the contribution 130 of wall emission and wall reflection to measured surface incident radiation, and to 131 propose a simple and convenient approach to estimate the magnitude of flame radiation.

132 CFD simulations of two different kinds of combustors are performed to validate the 133 theoretical analysis and the approach for flame radiation estimation. 


\section{2. Theoretical analysis}

138 A key assumption made in order to derive a simplified model of radiative heat transfer in

139 a combustion chamber is that the medium is gray. This assumption implies that the

140 radiative properties of the medium are independent of wavelength, which is a reasonable

141 assumption for flames involving suspended particles, like coal-fired flames, highly

142 luminous oil flames or some sooty gas-fired flames [33]. It could be less accurate in the

143 case of non-sooty gas-fired flames, where non-gray gaseous molecules such as $\mathrm{CO}_{2}$ and

$144 \mathrm{H}_{2} \mathrm{O}$ are the main contributors to radiation.

145

146 A simple axisymmetric combustor geometry as shown in Fig. 1 is used here to illustrate

147 the theoretical model. More general geoemtres will be discussed later. It is assumed that

148 the gas/particle medium in the combustor can be interpreted as a diffuse, gray surface that

149 is close to the wall such that the areas of this fictitious surafce and the wall are

150 approximately equal. The dotted line in Fig. 2 represents the fictitious surface, which is

151 defined to emit the same radiation power as the flame radiation, and has the same

152 emissvity as that of the medium. The emissivity of the fictitious surface, $\varepsilon_{s}$, is equivalent

153 to the emissivity of the medium, $\varepsilon_{m}$, eff. The flame radiation received by the wall (on a unit

154 area basis) can be expressed as

$155 E_{\text {flame }}=\varepsilon_{m, \text { eff }} \sigma T_{m, \text { eff }}^{4}$,

156 where $T_{m \text {, eff }}$ is the effective temperature of the medium, and $\sigma$ is the Stefan-Boltzmann

157 constant $\left(5.67 \times 10^{-8} \mathrm{~W} \cdot \mathrm{m}^{-2} \cdot \mathrm{K}^{-4}\right)$. Since the fictitious surface emit the same radiation

158 power as the flame radiation, the temperautre of the fictious surface, $T_{s}$, should be equal 159 to the effective temperature of the medium. 
161 For this analysis, the radiative properties of the wall are assumed to be diffuse and gray.

162 If the temperature and emissivity of the wall are $T_{w}$ and $\varepsilon_{w}$, respectively, the net radiative

163 heat flux $\left(\mathrm{W} / \mathrm{m}^{2}\right)$ transferred through the wall can be expressed by [34]

$164 \quad q_{n e t}=\frac{\sigma\left(T_{m, e f f}^{4}-T_{w}^{4}\right)}{\frac{1}{\varepsilon_{m, e f f}}+\frac{1}{\varepsilon_{w}}-1}$.

165

166 According to Kirchoff's law for diffuse, gray systems, the absorptivity of the wall is

167 equal to the emissivity of the wall, so the net radiative heat flux, $q_{\text {net }}$, is related to the 168 surface incident radiation on the wall, $G_{t o t a l}$, by the following:

$169 q_{\text {net }}=\varepsilon_{w} G_{\text {total }}-\varepsilon_{w} \sigma T_{w}^{4}$.

170 By combining Eq. (3) and (4) and rearranging, the surface incident radiation can be 171 expressed as

$172 \quad G_{\text {total }}=\frac{\sigma\left(T_{m, e f f}^{4}-T_{w}^{4}\right)}{\frac{\varepsilon_{w}}{\varepsilon_{m, e f f}}+1-\varepsilon_{w}}+\sigma T_{w}^{4}$.

173 As mentioned above, $G_{\text {total }}$ is the value obtained by a radiometer and is the combination

174 of flame radiation, $E_{\text {flame}}$, and the contribution of the walls, $G_{\text {wall }}$. Therefore, $G_{\text {wall }}$ can be

175 written as

$176 G_{\text {wall }}=G_{\text {total }}-E_{\text {flame }}=\frac{\sigma\left(T_{m, \text { eff }}^{4}-T_{w}^{4}\right)}{\varepsilon_{w}\left(\frac{1}{\varepsilon_{m, e f f}}-1\right)+1}+\sigma T_{w}^{4}-\varepsilon_{m, \text { eff }} \sigma T_{m, \text { eff }}^{4}$.

177 The contribution of the reflection of flame emission on $G_{\text {wall }}$ can be obtained by setting 178 the wall temperature $T_{w}$ to $0 \mathrm{~K}$, such that: 
$179 G_{\text {wall, reflect }}=\varepsilon_{m, \text { eff }} \sigma T_{m, \text { eff }}^{4} \frac{\left(1-\varepsilon_{w}\right)\left(1-\varepsilon_{m, \text { eff }}\right)}{\varepsilon_{w}+\left(1-\varepsilon_{w}\right) \varepsilon_{m, \text { eff }}}$.

180 The remaining part of $G_{\text {wall }}$ is a combination of two factors: (1) wall emission that comes

181 directly from the wall; and (2) wall emission that comes after being reflected off the wall

182 at least once. Both factors are considered as the contribution of wall emission in this work,

183 which can be expressed as

$184 G_{\text {wall, emission }}=G_{\text {wall }}-G_{\text {wall, reffect }}=\varepsilon_{w} \sigma T_{w}^{4} \frac{1-\varepsilon_{m, \text { eff }}}{\varepsilon_{w}+\left(1-\varepsilon_{w}\right) \varepsilon_{m, \text { eff }}}$.

186 From Eq. (7) and Eq. (8), as the wall temperature, $T_{w}$, increases, $G_{\text {wall,emission }}$ increases, but

$187 G_{\text {wall,reflect }}$ remains the same, indicating that $G_{\text {wall }}$ increases with $T_{\mathrm{w}}$. Equation (6) reveals

188 that $G_{\text {wall }}$ decreases as the wall emissivity $\varepsilon_{w}$ increases, because $\left(\frac{1}{\varepsilon_{m, \text { eff }}}-1\right)$ is always

189 greater than 0. Based on these findings, it is evident that the influence of the wall

190 (reflection and radiation) on the measurement of flame radiation can be minimized if the

191 wall surface is cold and non-reflective (i.e., $\varepsilon_{w}=1$ ). However, for most practical

192 combustion applications, a low wall temperature and low wall reflectivity usually do not

193 exist at the same time. Typically, there are two types of walls for combustion chambers:

194 refractory-lined walls and water-cooled metal walls [35]. Although refractory-lined walls

195 generally have high emissivity (i.e., low reflectivity), they also typically have large

196 thermal resistance (i.e., low conductivity and large thickness), which means the fire-side

197 surface of the wall becomes hot during operation. On the other hand, water-cooled walls

198 usually have a relatively low surface temperature, but the water tube materials generally

199 have lower emissivities than refractory materials. If there are ash/slag deposits on the 
200 water tubes, the emissivity of surface of the wall can vary from 0.4 to 0.9 depending on

201 the temperature and characteristics of the ash/slag deposits [36], but the surface

202 temperature of the wall will also increase due to the increased thermal resistance caused

203 by the deposits.

204

205 Table 1 quantifies the effect of wall conditions on the measurement of flame radiation.

206 Nominal properties of a lab-scale air-fired flame combusted under atmospheric pressure

207 are used, assuming $T_{m, e f f}=1500 \mathrm{~K}$ and $\varepsilon_{m, \text { eff }}=0.1$. Both a refractory-lined wall and a

208 water-cooled wall are considered. The temperature and emissivity of these two types of

209 walls are based on typical values reported in the literature [13].

210

211 Table 1 shows that, for the given flame and wall properties, the contribution of wall to the

212 measured surface incident radiation can be up to $56 \%$ for the refractory-lined wall and

$21340 \%$ for the water-cooled wall. For the refractory-lined wall, the major contribution of

214 the wall is from wall emission since the high wall temperature results in high radiation

215 power. For the water-cooled wall, the contribution of wall emission is smaller, but the

216 contribution of wall reflection is as high as $34 \%$, equivalent to $56 \%$ of the flame radiation.

217 Even if the emissivity of the water-cooled wall is increased to 0.8 , the total contribution 218 of the wall is still as high as $25 \%$, equivalent to $33 \%$ of the flame radiation, and $67 \%$ of

219 this contribution is from wall reflection. This example illustrates that the contribution of

220 the wall to the measured surface incident radiation is significant for both refractory-lined

221 walls and water-cooled walls. If the measured surface incident radiation is used as an 
222 indicator of flame radiation, the radiative characteristics of the flame can be grossly

223 underestimated.

\section{3. An approach to estimate flame radiation}

226 Since, when surface incident radiation is measured in practical combustion chambers the

227 contribution of the wall can be substantial compared with the flame radiation, it is 228 important to be able to deduce flame radiation from these measurements. Equation (5)

229 indicates that $G_{\text {total }}$ is a function of $T_{m, \text { eff }}$ and $\varepsilon_{m, \text { eff }}$ for given wall boundary conditions 230 (i.e., $T_{w}$ and $\varepsilon_{w}$ ). Theoretically, if the wall boundary conditions change slightly, $G_{\text {total }}$ 231 would change, but $T_{m, e f f}$ and $\varepsilon_{m, \text { eff }}$ would remain the same. Then, with two sets of 232 measurement data, the two unknowns, $T_{m, \text { eff }}$ and $\varepsilon_{m, \text { eff, }}$, can be solved, and then the flame 233 radiation can be calculated by Eq. (2). However, this approach requires the ability to 234 manually vary the wall conditions and accurately determine the surface temperature and 235 emissivity of the wall. While the former is possible for chambers with water-cooled walls,

236 the latter is sometimes challenging because deposits can affect the surface properties of 237 the wall.

239 In this section, an approach is proposed to estimate flame radiation without a prior 240 knowledge of the surface conditions of the wall. This approach is based on measurements 241 of the incident radiation and net radiative heat flux at the surface of the wall. The surface

242 incident radiation can be measured directly with a radiometer, like the ellipsoidal

243 radiometer used in [29]. The net radiative heat flux can be obtained by subtracting the 244 convective heat flux from the total heat flux through the wall. Typically, the total heat 
245 flux can be measured by a heat flux meter, like the disk-type heat flux meter used in [37].

246 The convective heat flux can be obtained by measuring or calculating the convective heat

247 transfer coefficient [3]. In some circumstances where radiation dominates heat transfer,

248 for example, in some oxygen-enriched solid-fuel combustors, convective heat flux may

249 even be neglected, and the total heat flux through the wall can simply represent the net 250 radiative heat flux.

251

252 Dividing Eq. (2) by Eq. (3) gives the ratio of the flame radiation to the net radiative heat 253 flux:

$254 \quad \frac{E_{\text {flame }}}{q_{\text {net }}}=\frac{1+\varepsilon_{m, \text { eff }}\left(\frac{1}{\varepsilon_{w}}-1\right)}{1-\left(\frac{T_{w}}{T_{m, e f f}}\right)^{4}}$.

255 If $T_{\mathrm{w}}{ }^{4}<<T_{m, e f f}{ }^{4}$, Eq. (8) becomes

$256 \frac{E_{\text {flame }}}{q_{\text {net }}} \approx 1+\varepsilon_{m, \text { eff }}\left(\frac{1}{\varepsilon_{w}}-1\right)>1$.

257 Based on Eq. (10), $E_{\text {flame }}$ is always larger than $q_{\text {net }}$, and the difference between $E_{\text {flame }}$ and

$258 q_{\text {net }}$ decreases as $\varepsilon_{m, e f f}$ decreases, given a constant wall emissivity. If $\varepsilon_{m, \text { eff }}$ is very small

259 (e.g., the medium is optically thin), then

$260 q_{\text {net }} \approx E_{\text {flame }}$.

261 Hence, the net radiative heat flux can be used to approximate flame radiation if the wall 262 surface temperature is relatively low compared with the medium temperature and the 263 medium is optically thin. Optically thin systems include most lab-scale and some pilot264 scale combustion facilities operating under atmospheric pressure. It follows from Eq. (10) 
265 that the accuracy of this approximation decreases with increasing optical thickness of the

266 medium and decreasing wall emissivity.

268 By dividing Eq. (2) by Eq. (5), the ratio of the flame radiation to the surface incident 269 radiation can be obtained:

$270 \quad \frac{E_{\text {flame }}}{G_{\text {total }}}=\frac{\varepsilon_{w}+\varepsilon_{m, \text { eff }}\left(1-\varepsilon_{w}\right)}{1+\varepsilon_{w}\left(\frac{1}{\varepsilon_{m, \text { eff }}}-1\right)\left(\frac{T_{w}}{T_{m, \text { eff }}}\right)^{4}}$.

271 If $T_{\mathrm{w}}{ }^{4}<<T_{m, \text { eff }}{ }^{4}$, Eq. (12) becomes

$272 \quad \frac{E_{\text {flame }}}{G_{\text {total }}} \approx \varepsilon_{w}+\varepsilon_{m, \text { eff }}\left(1-\varepsilon_{w}\right)<1$.

273 For those gas-fired systems where flame radiation mainly comes from soot emission, 274 scattering can be neglected due to the small size of the gas molecules and soot particles.

275 So if the medium is optically thick, the effective emissivity, $\varepsilon_{m, e f f}$, would be close to 1.0.

276 In coal-fired systems, char and ash particles can also be large contributors to radiation.

277 Since the particles in pulverized coal combustion boilers are large compared with the

278 wavelength of the radiation emitted at flame temperatures, the local particle absorption

279 coefficient $\alpha_{p}$ and scattering coefficient $\sigma_{p}$ can be respectively evaluated as [38, 39]:

$280 \quad \alpha_{p}=\sum_{i} \varepsilon_{p, i} n_{i} A_{p, i}$,

$281 \sigma_{p}=\sum_{i}\left(1-f_{p, i}\right)\left(1-\varepsilon_{p, i}\right) n_{i} A_{p, i}$.

282 Here, $i$ is the index number of a group of particles with the same size. Further, $\varepsilon_{p, i}, n_{i}, A_{p, i}$ 283 and $f_{p, i}$ are particle emissivity, number density, projected area, and scattering factor of the 284 ith group of particles, respectively. Particle emissivity, $\varepsilon_{p, i}$, in pulverized coal combustion 285 is generally believed to vary between the emissivity of unburned coal (1.0) and that of fly 
ash (0.6) [5]. The particle scattering factor, $f_{p, i}$, indicates the scattering properties of a

287 particle; a value of 1.0 indicates total forward scattering (effectively treated as

288 transmission). Generally, values from 0.6 to 0.9 were used for the particle scattering

289 factor in coal combustion processes [5, 40]. Thus, the value of $\left(1-f_{p, i}\right)\left(1-\varepsilon_{p, i}\right)$ lies in the

290 range of $0 \sim 0.016$, which indicates that $\sigma_{p}$, is much smaller than $\alpha_{p}$, in coal combustion

291 chambers. Therefore, the effective emissivity of the medium, $\varepsilon_{m, e f f}$, can also be expected

292 to be close to 1.0 when the medium is optically dense.

294 Based on Eq. (13), $E_{\text {flame }}$ is always smaller than $G_{\text {total }}$, and the difference between them

295 decreases as $\varepsilon_{m, \text { eff }}$ increases, given a constant wall emissivity. If $\varepsilon_{m, \text { eff }}$ is close to 1.0 , then

$296 G_{\text {total }} \approx E_{\text {flame }}$,

297 which indicates that the contribution of the wall to the measurement of flame radiation

298 becomes negligible, and the measured surface incident radiation can be directly used to

299 approximate flame radiation. Optically dense systems include pilot- and large-scale coal

300 combustion systems operating under elevated pressure [10, 23, 41]. Since the particle

301 number density almost increases proportionally with pressure for a given thermal input

302 and stoichiometric ratio, the optical thickness is also approximately proportional to 303 pressure.

304

305 Eq. (10) and (13) shows that $E_{\text {flame }}$ lies between $q_{\text {net }}$ and $G_{\text {total }}$. As $\varepsilon_{m, \text { eff }}$ increases from 0 to $3061, E_{\text {flame }}$ shifts from $q_{\text {net }}$ to $G_{\text {total }}$. It should be noted that, for a combustor geometry other 307 than the one shown in Fig. 1 (e.g. rectangular), the measured $q_{\text {net }}$ and $G_{\text {total }}$ could be non308 uniform along the perimeter of the chamber. In this case, the average value of $q_{\text {net }}$ and 
$309 G_{\text {total }}$ along the perimeter of the chamber should be used in the above approximation.

310 Also, for chambers with hot walls at a steady state (i.e. refractory-lined walls),

311 measurements of flame radiation can be conducted before a steady-state condition is

312 achieved (i.e., during start-up stage) so that the "cold wall” condition can still be satisfied.

313 Due to the heat capacity of the refractory walls, the change of the net radiative heat flux

314 at the surface of the wall should be small in the short period during start-up. In reality,

$315 q_{\text {net }}$ should be measured as close to the inner surface of the wall as possible to minimize

316 measurement error.

318 The above analysis provides a very convenient approach to estimate the flame radiation

319 for optically thin and optically dense systems without prior knowledge of the surface

320 properties of the wall. For systems with an optically thickness between optically thin and

321 optically dense, more complicated approaches have to be used and the surface properties

322 of the wall have to be known.

323

324 4. Validation by computational fluid dynamics (CFD) simulation

325 To validate the theoretical analysis and the approach to estimating flame radiation, CFD

326 simulations were performed on two coal-fired combustors: (1) a $19.2 \mathrm{kWth}$, atmospheric

327 pressure combustor, chosen to represent optically thin conditions; and (2) a 120 MWth,

328 50-atm pressurized combustor, chosen to represent optically dense conditions. The lab-

329 scale atmospheric pressure combustor is a simple 2-meter cylinder with a diameter of 0.2

$330 \mathrm{~m}$, as depicted in Figure 3. The inlet of the combustor is split into two streams. A central

331 high-speed air stream at $1500 \mathrm{~K}$ enters at $10 \mathrm{~m} / \mathrm{s}$. The surrounding air stream at $300 \mathrm{~K}$ 
332 enters at $0.6 \mathrm{~m} / \mathrm{s}$. Coal particles enter the furnace near the center of the combustor with a

333 mass flow rate of $0.0008 \mathrm{~kg} / \mathrm{s}$.

335 The large-scale pressurized combustor is a scale-up version of the lab-scale combustor, 336 with a 10-meter length and a 1-meter diameter, and is operated at $50 \mathrm{~atm}$. The residence

337 time of this large-scale combustor is the same as that of the lab-scale combustor, meaning 338 that the velocities of the air streams are five times larger than those of the lab-scale 339 combustor (i.e. $50 \mathrm{~m} / \mathrm{s}$ for the central stream and $3 \mathrm{~m} / \mathrm{s}$ for the surrounding stream). To

340 keep the stoichiometric ratio the same, the mass flow rate of the coal particles in the

341 large-scale combustor becomes $5 \mathrm{~kg} / \mathrm{s}$, which is 6250 times as large as that in the lab-

342 scale combustor. Since the gas volume flow rate is increased only by a factor of 125 , the

343 average particle number concentration of the large-scale combustor increases by a factor

344 of 50 compared to that of the lab-scale combustor. Hence the average particle extinction

345 coefficient also increases by a factor of 50 . The optical thickness from the centerline to

346 the wall, which equals the average particle extinction coefficient multiplied by the

347 distance between the centerline and the wall, will increase by a factor 250 compared to

348 that of the lab-scale combustor, leading to an optically dense system. The idea of utilizing

349 pressurization to create optically dense media has also been found in [9, 10]. 350

351 ANSYS FLUENT 13.0 was used for simulating the coal combustion processes in the two 352 combustors. The flow field was modeled applying the Reynolds Averaged Navier Stokes 353 (RANS) equations with the Semi-Implicit Method for Pressure Linked Equations 354 (SIMPLE) algorithm to address the pressure-velocity coupling. The shear stress transport 
355 (SST) $k-\omega$ model [42] and the Eddy Dissipation Model (EDM) [43, 44] were utilized

356 for modeling of turbulence and turbulence-chemistry interactions. A simple single-rate

357 devolatilization model and the kinetics/diffusion-limited char combustion model were

358 employed since they are not the focus of this work. The radiative transport equation was

359 solved by the Discrete Ordinates (DO) [45, 46] model, which can be used for both

360 optically thin and optically dense media. Each octant of the entire angular space $4 \pi$ at any

361 spatial location was discretized into $5 \times 5$ solid angles (i.e. control angles), leading to a

362 total of 200 directions. Further refined discretization did not show any noticeable

363 improvement in terms of the distributions of temperature and incident radiation. Gaseous

364 radiation and absorption was considered with the domain-based Weighted Sum of Gray

365 Gases model (WSGGM). The combustor wall was assumed to be gray and diffuse. The

366 particle emissivity and particle scattering factor were both set to be 0.9 [5, 47].

368 4.1. Lab-scale atmospheric pressure combustor

369 To demonstrate the effects of wall emission and wall refection on the radiative heat

370 transfer in the lab-scale combustor, four types of wall boundary conditions were

371 employed in the simulation, as shown in Table 2. In Case 1, the wall temperature was set

372 to room temperature $298 \mathrm{~K}$ and the wall emissivity was set to 1.0 . Under these conditions,

373 the incident radiation on the wall is equivalent to the flame radiation measured from a

374 radiometer in an open atmospheric environment. This incident radiation should be very

375 close to the actual flame radiation (i.e. $E_{\text {flame }}=\varepsilon_{m, \text { eff }} \sigma T_{m, \text { eff }}^{4}$ ). 
377 Figure 4 shows the predicted temperature contours in the lab-scale combustor for

378 different wall conditions. Even though the heat transfer rate to the wall is quite different

379 in these cases, in the combustion zone (i.e., $x \approx 0-0.4 \mathrm{~m}$, where $x$ is the distance from the

380 inlet), the flame shapes and flame temperatures among the different cases are very close.

381 Varied wall boundary conditions have little impact on the flame in this combustor.

383 Figure 5 shows the radial profiles of temperature and total extinction coefficient (i.e., gas

384 extinction coefficient plus particle extinction coefficient) for all cases at $x=0.4 \mathrm{~m}$. By

385 integrating the extinction coefficient profiles along the radial distance, the optical

386 thickness of the medium from the centerline to the wall (i.e., the area below the extinction

387 coefficient profile) can be obtained. The calculated optical thickness is around 0.06.

388 Based on Beer's law, this optical thickness corresponds to a transmissivity of $94 \%$, which

389 means that $94 \%$ of the radiation originating from the centerline can pass through the

390 medium. Obviously, the medium in the combustor is optically thin.

392 The radiation from an optically thin medium mainly originates from local regions where

393 the temperature and absorption coefficient are both high [10]. For the lab-scale

394 combustor, the absorption coefficient is close to the extinction coefficient, as mentioned

395 above. As shown in Figure 5, all cases have identical extinction coefficient profiles and

396 nearly identical temperature profiles, except that the near-wall temperatures (between

3970.04 and $0.05 \mathrm{~m}$ ) are different due to different wall boundary conditions. While the

398 temperature in this region varies between the cases, the emission in this region is

399 negligible for all cases due to the low absorption coefficient. Thus, it can be reasonably 
400 considered that the four cases have the same magnitudes of flame radiation. This

401 assumption allows us to investigate the effects of wall emission and wall reflection with

402 the same flame characteristics.

403

404 Table 3 shows the predicted surface incident radiation, $G_{\text {total }}$, and the net radiative wall

405 heat flux, $q_{\text {net }}$, at $x=0.4 \mathrm{~m}$ on the wall from the CFD model. As mentioned above, for

406 optically thin systems, if the wall temperature is relatively low, $E_{\text {flame }}$ can be

407 approximated by $q_{\text {net. }}$ In Table 3, the measured $G_{\text {total }}$ of Case 1 (i.e. cold and black wall)

408 is expected to be close to the flame radiation. It can be found that the $q_{\text {net }}$ of Case 3,

409 where the wall temperature is relatively low, agrees reasonably well (around 13\% error)

410 with the $G_{\text {total }}$ of Case 1, while the $G_{\text {total }}$ of Case 3, and both the $q_{\text {net }}$ and $G_{\text {total }}$ of Case 2

411 and Case 4 differ significantly with the $G_{\text {total }}$ of Case 1 . Later we will show that,

412 compared with the actual flame radiation, the net radiative heat flux in Case 3 differs

413 even less.

414

415 It was mentioned earlier that, if the flame properties are not affected by the wall 416 conditions, then with two sets of wall conditions and associated $G_{\text {total }}$ or $q_{\text {net }}$, it is possible

417 to solve for $T_{m, \text { eff }}$ and $\varepsilon_{m, \text { eff. }}$ Substituting $T_{w}=298 \mathrm{~K}, \varepsilon_{w}=1.0, G_{\text {total }}=6.196 \mathrm{~kW} / \mathrm{m}^{2}$ and

$418 T_{\mathrm{w}}=900 \mathrm{~K}, \varepsilon_{\mathrm{w}}=0.4, G_{\text {total }}=42.363 \mathrm{~kW} / \mathrm{m}^{2}$ into Eq. (5), and the effective temperature

419 and emissivity of the medium can be solved for: $T_{m, \text { eff }}=1025.3 \mathrm{~K}, \varepsilon_{m, \text { eff }}=0.092$. It should

420 be noted that using any two of the above cases gives very similar results. The flame

421 radiation can be obtained based on these two parameters: $E_{\text {flame }}=\varepsilon_{m, \text { eff }} \sigma T_{m, \text { eff }}^{4}=5.791$

$422 \mathrm{~kW} / \mathrm{m}^{2}$. Because this flame radiation is very small, even the wall emission at room 
423 temperature can have a noticeable effect on the incident radiation. The $G_{\text {total }}$ measured in

424 Case 1 differs by $7 \%$ compared with this $E_{\text {flame }}$. However, the $q_{\text {net }}$ in Case 1 only differs

425 by $0.7 \%$. In Case $3\left(T_{\mathrm{w}}=500 \mathrm{~K}, \varepsilon_{\mathrm{w}}=0.8\right)$, the $G_{\text {total }}$ differs by $77 \%$ compared with the

$426 E_{\text {flame }}$ and the $q_{\text {net }}$ only differs by $7 \%$.

428 More cases were simulated, in which the wall temperature was set to $500 \mathrm{~K}$ and the wall 429 emissivity was varied stepwise from 0.8 to 0.2 . Table 4 shows the $G_{\text {total }}$, $q_{\text {net }}$, and the 430 relative differences between the $q_{\text {net }}$ and $E_{\text {flame }}$ at $x=0.4 \mathrm{~m}$. As wall emissivity decreases, $431 G_{\text {total }}$ changes substantially, but the change of $q_{\text {net }}$ is relatively small. The relative 432 difference between $q_{\text {net }}$ and $E_{\text {flame }}$ increases as the wall emissivity decreases, in 433 accordance with Eq. (10). As long as the wall emissivity is larger than 0.6, which is true 434 for most combustion chambers, the relative difference remains lower than 10\%. For 435 comparison, Table 4 also provides the relative errors for the traditional approach of 436 obtaining the flame radiation by measuring the difference of incident radiation with and 437 without the flame [12]. In this approach, only the impact of wall emission has been 438 considered, and the flame radiation is given by $\left(G_{t o t a l}-\sigma T_{w}{ }^{4}\right)$. Clearly, for optically thin 439 systems, the proposed approach in this study has a much better accuracy than the 440 previous approach.

442 With the determined $T_{m, \text { eff }}$ and $\varepsilon_{m}$, eff, $G_{\text {total }}$ and $q_{\text {net }}$ for any wall conditions can also be 443 calculated based on the theoretical model in Section 2. Figure 6 shows the $G_{\text {total }}$ and $q_{\text {net }}$ 444 predicted by the theoretical model in Section 2 using $T_{m, \text { eff }}=1025.3 \mathrm{~K}$ and $\varepsilon_{m, \text { eff }}=0.092$, 445 together with the CFD simulation results. The results of the two models agree very well. 
446 The flame radiation, $E_{\text {flame, }}$, is the incident radiation (or net radiative heat flux) at $T_{\mathrm{w}}=0 \mathrm{~K}$,

447 which is also showed in Fig. 6 together with $\pm 25 \%$ boundary lines (dashed lines). Note

448 that Fig. 6a and Fig. 6b have different vertical scales, the solid lines for $q_{\text {net }}$ are much

449 tighter to each other compared with those for $G_{\text {total }}$. It is found that the $G_{\text {total }}$ always

450 deviates from $E_{\text {flame }}$ by more than $25 \%$ when the wall emissivity is lower than 0.7 or the

451 wall temperature is higher than $420 \mathrm{~K}$, while the $q_{\text {net }}$ always deviates from $E_{\text {flame }}$ by more

452 than $25 \%$ when the wall emissivity is lower than 0.2 or the wall temperature is higher 453 than $750 \mathrm{~K}$.

454

455 4.2. Large-scale pressurized combustor

456 The same simulations as shown in Table 2 were also carried out for the pressurized

457 combustor. Figure 7 shows the temperature contours in the pressurized combustor for all

458 these cases. Similar to the lab-scale combustor, the overall flame shapes and temperature

459 distributions are insensitive to the wall boundary conditions.

461 Figure 8 shows the radial profiles of temperature and total extinction coefficient at $x=2$

$462 \mathrm{~m}$. Again, the extinction coefficients and temperatures for these cases are almost identical.

463 There are differences only in the temperatures in the near-wall region. The calculated 464 total optical thicknesses from the centerline to the wall based on the extinction coefficient 465 profiles are as large as 6.2, which corresponds to a transmissivity of 0.002 , indicating that 466 the medium is optically dense. In an optically dense medium, radiation on the wall should 467 be determined by local conditions [10]. However, in this case shown in Fig.8, even 468 though the optical thickness from the centerline to the wall is large, the optical thickness 
469 in the near-wall region is still very small, due to the low extinction coefficient near the

470 wall. From $y=0.2$ ( $y$ is the radial distance from the centerline) to the wall, the optical

471 thickness is only 0.88 , corresponding to a transmissivity of 0.41 . Hence $41 \%$ of the

472 incident radiation at $y=0.2$ can still reach the wall. Therefore, it can be assumed that the

473 temperature difference in the very near-wall region will not cause substantial change in

474 the flame radiation, meaning the four cases still have the same magnitudes of flame 475 radiation.

476

477 Table 5 shows the $G_{\text {total }}$ and $q_{\text {net }}$ for all cases at $x=2 \mathrm{~m}$. Substituting $T_{w}=298 \mathrm{~K}, \varepsilon_{w}=1.0$, $478 G_{\text {total }}=125.52 \mathrm{~kW} / \mathrm{m}^{2}$ and $T_{w}=900 \mathrm{~K}, \varepsilon_{w}=0.4, G_{\text {total }}=138.26 \mathrm{~kW} / \mathrm{m}^{2}$ into Eq. (5), and 479 the effective temperature and emissivity of the medium can be solved for: $T_{m, \text { eff }}=1263.2$ $480 \mathrm{~K}, \varepsilon_{m, \text { eff }}=0.869$. The $E_{\text {flame }}$ obtained from these two parameters is $125.46 \mathrm{~kW} / \mathrm{m}^{2}$. The $481 G_{\text {total }}$ in Case $3\left(T_{\mathrm{w}}=500 \mathrm{~K}, \varepsilon_{\mathrm{w}}=0.8\right)$ differs only by $1.2 \%$ compared with the $E_{\text {flame }}$ but 482 the $q_{\text {net }}$ differs by $21 \%$, demonstrating that for an optically dense system, $G_{\text {total }}$ becomes a 483 better approximation for the flame radiation.

485 Cases with various wall emissivities but a constant wall temperature at $500 \mathrm{~K}$ are shown 486 in Table 6. As opposed to the cases for the lab-scale combustor, this time $q_{\text {net }}$ changes 487 substantially with the wall emissivity but the changes in $G_{\text {total }}$ is small. The relative 488 difference between the $G_{\text {total }}$ and $E_{\text {flame }}$ increases with decreasing wall emissivity, 489 following Eq. (12). The relative differences remain lower than $10 \%$ even when the wall 490 emissivity is as low as 0.4. Unlike the cases for the lab-scale combustor, here the present 491 estimation approach has a very similar accuracy compared with the traditional approach 
492 which accounts for the impact of only wall emission. For relatively high wall emissivities,

493 the present approach has a slightly better accuracy, while for relatively low wall

494 emissivities, the traditional approach has a slightly better accuracy.

495

496 Figure 9 shows a comparison between the results of the theoretical radiation model using

497 flame properties of $T_{m, e f f}=1263.2 \mathrm{~K}$ and $\varepsilon_{m, e f f}=0.869$, and the results of the CFD model.

498 As can be seen, they agree very well. In contrast with the lab-scale combustor, the solid

499 lines for $G_{\text {total }}$ are much tighter to each other compared with those for $q_{\text {net }}$. It is found that

500 in the entire range of the wall temperature investigated, the $G_{\text {total }}$ never deviates from the

$501 E_{\text {flame }}$ by more than $25 \%$. The $q_{\text {net }}$ deviates from $E_{\text {flame }}$ by more than $25 \%$ when the wall

502 emissivity is lower than 0.7 or the wall temperature is higher than $900 \mathrm{~K}$.

503

\section{5. Conclusions}

505 In the present study, a theoretical analysis was conducted to evaluate the contribution of

506 the wall to the radiation values measured by a radiometer in a closed combustion

507 chamber. A simplified radiation model was developed by considering the non-

508 homogeneous gas/particle medium inside the chamber as a grey surface with uniform

509 properties which radiates with the same emissive power as the medium. Analyses show

510 that surface incident radiation measurements can be used to represent flame radiation

511 only when the wall is cold and black (i.e., emissivity equals 1). For a lab-scale

512 atmospheric pressure combustor the contribution of the wall to the measured incident

513 radiation can be as high as $56 \%$ for a typical refractory-lined wall, and as high as $40 \%$ for

514 a typical water-cooled wall. 
516 To distinguish the flame radiation from the measured incident radiation, an approach was

517 proposed to estimate the flame radiation for optically thin (most lab-scale and some pilot-

518 scale combustion facilities operating under atmospheric pressure) and optically dense

519 systems (pilot- and large-scale coal combustion systems operating under elevated

520 pressures). The main advantage of the approach is that it does not require prior

521 knowledge of the wall surface properties. The approach was based on measurements of

522 the net wall radiative heat flux and surface incident radiation on the wall. If the wall

523 temperature is much lower than the flame temperature, for optically thin systems, the net

524 radiative wall heat flux can be used to approximate the flame radiation; for optically

525 dense systems, the surface incident radiation can be used to approximate the flame

526 radiation. The analyses showed that the accuracy of the approximation approach

527 presented herein increases as the wall emissivity increases. CFD simulations were

528 performed for two combustors, one optically thin and one optically dense, to validate the

529 simplified radiation model and the flame radiation approximation approach. The CFD

530 model results agree well with the simplified flame radiation model results. For the

531 optically thin combustor, the error of the flame radiation approximation approach is less

532 than $10 \%$ when the wall emissivity value is greater than 0.6 , which is true for most

533 practical combustion chambers. For the optically dense combustor, the error of the

534 approximation approach is less than $10 \%$ when the wall emissivity value is greater than

$535 \quad 0.4$.

537 Acknowledgements 
538 This material is based upon work supported by the Department of Energy under Award

539 Number DE-FE0009702, by the Consortium for Clean Coal Utilization at Washington

540 University in St. Louis.

541

\section{Disclaimer}

543 This report was prepared as an account of work sponsored by an agency of the United

544 States Government. Neither the United States Government nor any agency thereof, nor

545 any of their employees, makes any warranty, express or implied, or assumes any legal

546 liability or responsibility for the accuracy, completeness, or usefulness of any information,

547 apparatus, product, or process disclosed, or represents that its use would not infringe

548 privately owned rights. Reference herein to any specific commercial product, process, or

549 service by trade name, trademark, manufacturer, or otherwise does not necessarily

550 constitute or imply its endorsement, recommendation, or favoring by the United States

551 Government or any agency thereof. The views and opinions of the authors expressed

552 herein do not necessarily state or reflect those of the United States Government or any

553 agency thereof.

554

555 Reference

556 [1] C. Tien, S. Lee, Flame radiation, Progress in Energy and Combustion Science, 8 557 (1982) 41-59.

558 [2] R. Viskanta, M. Mengüç, Radiation heat transfer in combustion systems, Progress in 559 Energy and Combustion Science, 13 (1987) 97-160.

560 [3] J. Smart, P. O’nions, G. Riley, Radiation and convective heat transfer, and burnout in 561 oxy-coal combustion, Fuel, 89 (2010) 2468-2476.

562 [4] J.P. Smart, R. Patel, G.S. Riley, Oxy-fuel combustion of coal and biomass, the effect 563 on radiative and convective heat transfer and burnout, Combustion and Flame, 157 (2010) $5642230-2240$. 
[5] C. Yin, On gas and particle radiation in pulverized fuel combustion furnaces, Applied 566 Energy, (2015).

567 [6] B.W. Butler, M.K. Denison, B.W. Webb, Radiation heat transfer in a laboratory-scale, pulverized coal-fired reactor, Experimental Thermal and Fluid Science, 9 (1994) 69-79. [7] B.W. Butler, B.W. Webb, Local temperature and wall radiant heat flux measurements in an industrial scale coal fired boiler, Fuel, 70 (1991) 1457-1464.

[8] S. Park, C. Tien, Radiation induced ignition of solid fuels, International journal of heat and mass transfer, 33 (1990) 1511-1520. [9] F. Xia, Z. Yang, A. Adeosun, A. Gopan, B.M. Kumfer, R.L. Axelbaum, Pressurized oxy-combustion with low flue gas recycle: Computational fluid dynamic simulations of radiant boilers, Fuel, 181 (2016) 1170-1178.

[10] F. Xia, Z. Yang, A. Adeosun, B.M. Kumfer, R.L. Axelbaum, Control of radiative heat transfer in high-temperature environments via radiative trapping-Part I: Theoretical analysis applied to pressurized oxy-combustion, Fuel, 172 (2016) 81-88.

[11] K. Zhou, N. Liu, L. Zhang, K. Satoh, Thermal Radiation from Fire Whirls: Revised Solid Flame Model, Fire Technol, 50 (2014) 1573-1587.

[12] D. Woycenko, W. Van de Kamp, P. Roberts, Combustion of pulverized coal in a mixture of oxygen and recycled flue gas. Summary of the APG research program, IFRF Doc, in, F98/Y/4. Ijmuiden, The Netherlands: International Flame Research Foundation (IFRF), 1995.

[13] K. Andersson, R. Johansson, S. Hjärtstam, F. Johnsson, B. Leckner, Radiation intensity of lignite-fired oxy-fuel flames, Experimental Thermal and Fluid Science, 33 (2008) 67-76.

[14] K. Andersson, F. Johnsson, Flame and radiation characteristics of gas-fired O 2/CO 2 combustion, Fuel, 86 (2007) 656-668.

[15] Y. Tan, M.A. Douglas, K.V. Thambimuthu, CO 2 capture using oxygen enhanced combustion strategies for natural gas power plants, Fuel, 81 (2002) 1007-1016.

[16] C. Lou, H.-C. Zhou, P.-F. Yu, Z.-W. Jiang, Measurements of the flame emissivity and radiative properties of particulate medium in pulverized-coal-fired boiler furnaces by image processing of visible radiation, Proceedings of the Combustion Institute, 31 (2007) 2771-2778.

[17] K. Andersson, R. Johansson, F. Johnsson, Thermal radiation in oxy-fuel flames, International Journal of Greenhouse Gas Control, 5 (2011) S58-S65.

[18] D. Bäckström, D. Gall, M. Pushp, R. Johansson, K. Andersson, J.B.C. Pettersson, Particle composition and size distribution in coal flames - The influence on radiative heat transfer, Experimental Thermal and Fluid Science, 64 (2015) 70-80.

[19] D. Bäckström, R. Johansson, K. Andersson, F. Johnsson, S. Clausen, A. Fateev, Measurement and Modeling of Particle Radiation in Coal Flames, Energy \& Fuels, 28 (2014) 2199-2210.

[20] E.S. Oran, J.P. Boris, Detailed modelling of combustion systems, Progress in Energy and Combustion Science, 7 (1981) 1-72.

[21] P. Nakod, G. Krishnamoorthy, M. Sami, S. Orsino, A comparative evaluation of gray and non-gray radiation modeling strategies in oxy-coal combustion simulations, Applied Thermal Engineering, 54 (2013) 422-432.

610 [22] A.A. Bhuiyan, J. Naser, Numerical modelling of oxy fuel combustion, the effect of radiative and convective heat transfer and burnout, Fuel, 139 (2015) 268-284. 
611 [23] F. Xia, Z. Yang, A. Adeosun, B.M. Kumfer, R.L. Axelbaum, Staged, pressurized 612 oxy-combustion: computational fluid dynamic simulations of radiant boilers, in: The 8th 613 International Symposium on Coal Combustion Beijng, China, 2015.

614 [24] J. Zhang, T. Ito, S. Ito, D. Riechelmann, T. Fujimori, Numerical investigation of 615 oxy-coal combustion in a large-scale furnace: Non-gray effect of gas and role of particle 616 radiation, Fuel, 139 (2015) 87-93.

617 [25] V. Becher, J.-P. Bohn, P. Dias, H. Spliethoff, Validation of spectral gas radiation 618 models under oxyfuel conditions - part B: natural gas flame experiments, International Journal of Greenhouse Gas Control, 5 (2011) S66-S75.

[26] R. Johansson, B. Leckner, K. Andersson, F. Johnsson, Influence of particle and gas radiation in oxy-fuel combustion, International Journal of Heat and Mass Transfer, 65 (2013) 143-152.

623 [27] A. Paist, A. Poobus, T. Tiikma, Probes for measuring heat transfer parameters and fouling intensity in boilers, Fuel, 81 (2002) 1811-1818. [28] Y. Tan, E. Croiset, M.A. Douglas, K.V. Thambimuthu, Combustion characteristics of coal in a mixture of oxygen and recycled flue gas, Fuel, 85 (2006) 507-512.

[29] B. Butler, M. Denison, B. Webb, Radiation heat transfer in a laboratory-scale, pulverized coal-fired reactor, Experimental Thermal and Fluid Science, 9 (1994) 69-79. [30] B. BrajuSkovid, N. Afgan, " CLEAN" TYPE HEAT FLUX METER, (1990).

632 [32] A.K. Chambers, J.R. Wynnyckyj, E. Rhodes, A Furnace Wall Ash Monitoring 633 System for Coal Fired Boilers, Journal of Engineering for Power, 103 (1981) 532-538.

634 [33] M.J. Yu, S.W. Baek, J.H. Park, An extension of the weighted sum of gray gases non635 gray gas radiation model to a two phase mixture of non-gray gas with particles, 636 International Journal of Heat and Mass Transfer, 43 (2000) 1699-1713.

637 [34] F.P. Incropera, Fundamentals of heat and mass transfer, John Wiley \& Sons, 2011.

638 [35] P. Basu, C. Kefa, L. Jestin, Boilers and burners: design and theory, Springer Science 639 \& Business Media, 2012.

640 [36] T. Wall, S. Bhattacharya, D. Zhang, R. Gupta, X. He, The properties and thermal 641 effects of ash deposits in coal-fired furnaces, Progress in Energy and Combustion Science, 64219 (1993) 487-504.

643 [37] A. Chambers, J. Wynnyckyj, E. Rhodes, A furnace wall ash monitoring system for 644 coal fired boilers, Journal of Engineering for Power, 103 (1981) 532-538.

645 [38] C. Yin, J. Yan, Oxy-fuel combustion of pulverized fuels: Combustion fundamentals 646 and modeling, Applied Energy, 162 (2016) 742-762.

647 [39] E. Chui, P.M. HUGHES, G. Raithby, Implementation of the finite volume method 648 for calculating radiative transfer in a pulverized fuel flame, Combustion Science and 649 Technology, 92 (1993) 225-242.

650 [40] L. Ma, M. Gharebaghi, R. Porter, M. Pourkashanian, J. Jones, A. Williams, 651 Modelling methods for co-fired pulverised fuel furnaces, Fuel, 88 (2009) 2448-2454.

652 [41] Z. Yang, F. Xia, A. Adeosun, B.M. Kumfer, R.L. Axelbaum, Control of radiative 653 heat transfer in pressurized, high temperature combustion applications, in: 9th U. S. 654 National Combustion Meeting, Cincinnati, Ohio, 2015.

655 [42] F.R. Menter, Two-equation eddy-viscosity turbulence models for engineering 656 applications, AIAA journal, 32 (1994) 1598-1605. 
657 [43] B.F. Magnussen, B.H. Hjertager, On mathematical modeling of turbulent 658 combustion with special emphasis on soot formation and combustion, Symposium 659 (International) on Combustion, 16 (1977) 719-729.

660 [44] D. Spalding, Mixing and chemical reaction in steady confined turbulent flames, in: 661 Symposium (International) on Combustion, Elsevier, 1971, pp. 649-657.

662 [45] E.H. Chui, G.D. Raithby, Computation of radiant heat transfer on a nonorthogonal 663 mesh using the finite-volume method, Numerical Heat Transfer, Part B: Fundamentals, 66423 (1993) 269-288.

665 [46] G.D. Raithby, E.H. Chui, A finite-volume method for predicting a radiant heat 666 transfer in enclosures with participating media, Journal of Heat Transfer, 112 (1990) 415667423.

668 [47] R. Backreedy, L. Fletcher, L. Ma, M. Pourkashanian, A. Williams, Modelling 669 pulverised coal combustion using a detailed coal combustion model, Combustion science 670 and technology, 178 (2006) 763-787.

671

672 


\section{$673 \quad$ Figure captions}

674 Figure 1. Illustration of the influence of walls on total incident radiation received by a 675 radiometer. Flame is depicted by red zone.

676 Figure 2. Simplified model to discribe radiative heat transfer inside a chamber.

677 Figure 3. Atmospheric pressure furnace with pulverized coal combustion.

678 Figure 4. Temperature contours $(\mathrm{K})$ in the lab-scale atmospheric pressure combustor.

679 Figure 5. Predicted radial profiles of temperature (left) and total extinction coefficient 680 (right) at $x=0.4 \mathrm{~m}$ in the lab-scale atmospheric pressure combustor.

681 Figure 6. (a) The incident radiation and (b) the net radiative heat flux predicted by the 682 theoretical model (solid lines) and the CFD model (symbols) for the lab-scale 683 atmospheric pressure coal combustor. The values in the parentheses indicate the 684 wall temperature (the first value) and wall emissivity (the second value) in the $685 \quad$ CFD simulations.

686 Figure 7. Temperature contours $(\mathrm{K})$ in the large-scale pressurized combustor for different $687 \quad$ cases.

688 Figure 8. Predicted radial profiles of temperature (left) and total extinction coefficient 689 (right) at $x=2 \mathrm{~m}$ in the large-scale pressurized combustor.

690 Figure 9. Wall incident radiation (left) and net radiative heat flux (right) predicted by the 691 simplified radiation model (lines) and the CFD model (symbols) for the 692 pressurized combustor. The values in the parentheses indicate the wall 693 temperature (the first value) and wall emissivity (the second value) in the CFD $694 \quad$ simulations. 
Table 1. The effects of walls on measurements of flame radiation.

\begin{tabular}{ccc}
\hline & Refractory-lined wall & Water-cooled wall \\
\hline Wall conditions & $T_{w}=900 \mathrm{~K}$ and $\varepsilon_{w}=0.9$ & $T_{w}=500 \mathrm{~K}$ and $\varepsilon_{w}=0.6$ \\
$E_{\text {flame }}\left(\mathrm{kW} / \mathrm{m}^{2}\right)$ & 28.70 & 28.70 \\
$G_{\text {total }}\left(\mathrm{kW} / \mathrm{m}^{2}\right)$ & 64.66 & 47.84 \\
$G_{\text {wall }} / G_{\text {total }}$ & $55.6 \%$ & $40 \%$ \\
$G_{\text {wall,emission }} / G_{\text {total }}$ & $51.2 \%$ & $6.3 \%$ \\
$G_{\text {wall, } \text { reflect }} / G_{\text {total }}$ & $4.4 \%$ & $33.7 \%$ \\
$\left(G_{\text {total }}-E_{\text {flame }}\right) / E_{\text {flame }}$ & $125 \%$ & $67 \%$ \\
\hline
\end{tabular}


Table 2. Wall boundary conditions for different simulation cases

\begin{tabular}{ccc}
\hline & Wall temperature (K) & Wall emissivity \\
\hline Case 1 & 298 & 1.0 \\
Case 2 & 900 & 0.8 \\
Case 3 & 500 & 0.8 \\
Case 4 & 900 & 0.4 \\
\hline
\end{tabular}


Table 3. Surface incident radiation and net radiative heat flux at $x=0.4 \mathrm{~m}$ on the wall in the lab-scale combustor

\begin{tabular}{llrlr}
\hline & Case 1 & Case 2 & Case 3 & Case 4 \\
\hline$G_{\text {total }}\left(\mathrm{kW} / \mathrm{m}^{2}\right)$ & 6.196 & 40.108 & 10.285 & 42.363 \\
$q_{\text {net }}\left(\mathrm{kW} / \mathrm{m}^{2}\right)$ & 5.749 & 2.328 & 5.393 & 2.066 \\
\hline
\end{tabular}


Table 4. Effects of the wall emissivity on the simulation results of the lab-scale combustor when the wall is at $500 \mathrm{~K}$

\begin{tabular}{ccccc}
\hline Wall emissivity & 0.8 & 0.6 & 0.4 & 0.2 \\
\hline$G_{\text {total }}\left(\mathrm{kW} / \mathrm{m}^{2}\right)$ & 10.285 & 12.235 & 15.674 & 23.232 \\
$q_{\text {net }}\left(\mathrm{kW} / \mathrm{m}^{2}\right)$ & 5.393 & 5.215 & 4.852 & 3.938 \\
$\left(q_{\text {net }}-E_{\text {flame }}\right) / E_{\text {flame }}(\%)$ & -6.9 & -9.9 & -16.2 & -32 \\
$\left(G_{\text {total }}-\sigma T_{w}{ }^{4}-E_{\text {flame }}\right) / E_{\text {flame }}(\%)$ & 16.4 & 50.1 & 109.5 & 240.0 \\
\hline
\end{tabular}


Table 5. Simulation results of the incident radiation and the net radiative heat flux at $x$ $=2 \mathrm{~m}$ on the wall in the large-scale pressurized combustor

\begin{tabular}{lllll}
\hline & Case 1 & Case 2 & Case 3 & Case 4 \\
\hline$G_{\text {total }}\left(\mathrm{kW} / \mathrm{m}^{2}\right)$ & 125.52 & 131.53 & 126.99 & 138.26 \\
$q_{\text {net }}\left(\mathrm{kW} / \mathrm{m}^{2}\right)$ & 125.07 & 75.67 & 98.76 & 40.42 \\
\hline
\end{tabular}


Table 6. Effect of wall emissivity on the simulation results of the large-scale pressurized combustor when the wall temperature is $500 \mathrm{~K}$.

\begin{tabular}{ccccc}
\hline Emissivity & 0.8 & 0.6 & 0.4 & 0.2 \\
\hline$q_{\text {net }}\left(\mathrm{kW} / \mathrm{m}^{2}\right)$ & 98.76 & 77.33 & 52.71 & 27.51 \\
$G_{\text {total }}\left(\mathrm{kW} / \mathrm{m}^{2}\right)$ & 126.99 & 132.42 & 135.32 & 141.10 \\
$\left(G_{\text {total }}-E_{\text {flame }}\right) / E_{\text {flame }}(\%)$ & 1.2 & 5.5 & 7.8 & 12.5 \\
$\left(G_{\text {total }} \sigma T_{w}{ }^{4}-E_{\text {flame }}\right) / E_{\text {flame }}(\%)$ & -1.6 & 2.7 & 5.0 & 9.6 \\
\hline
\end{tabular}




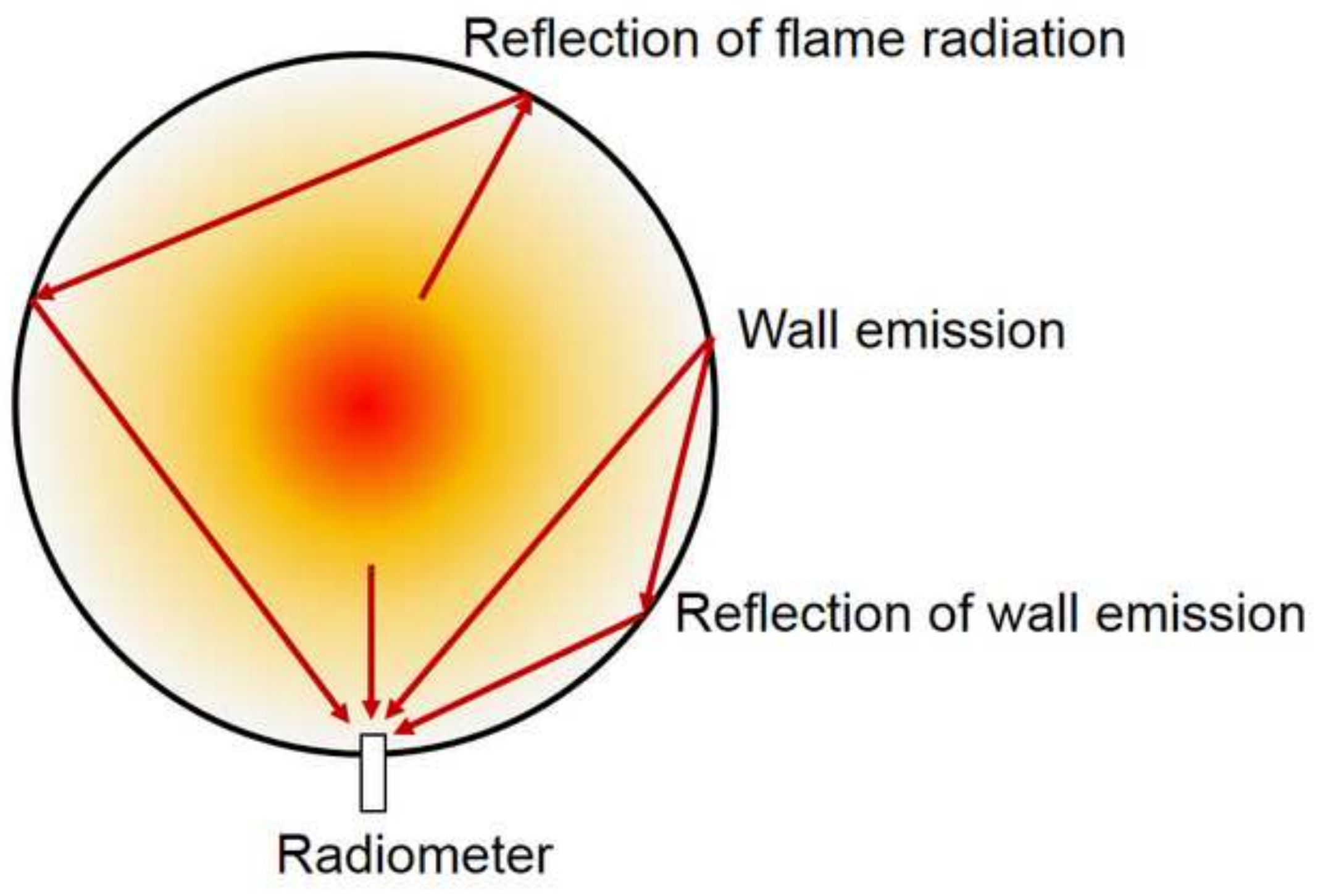




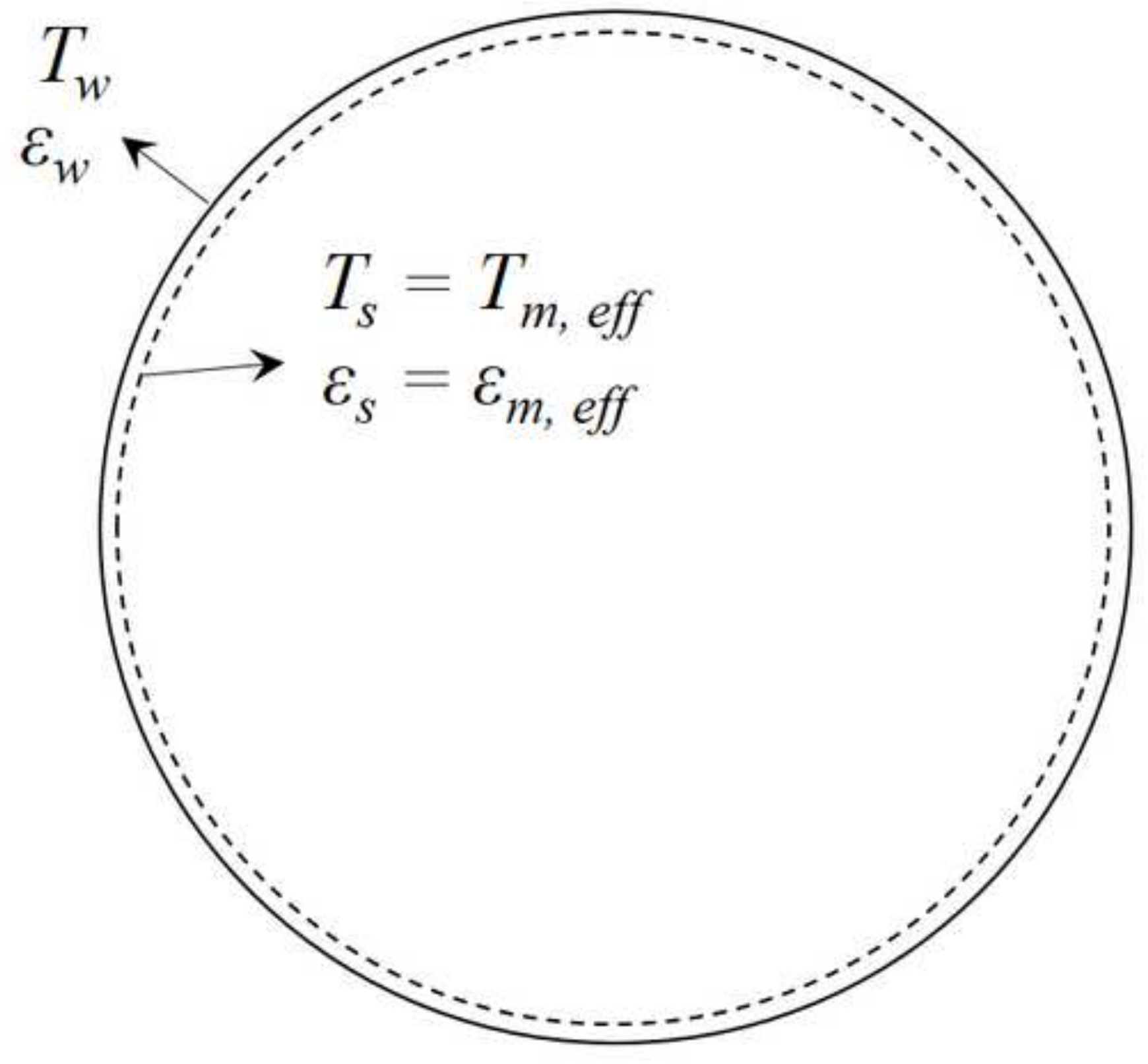




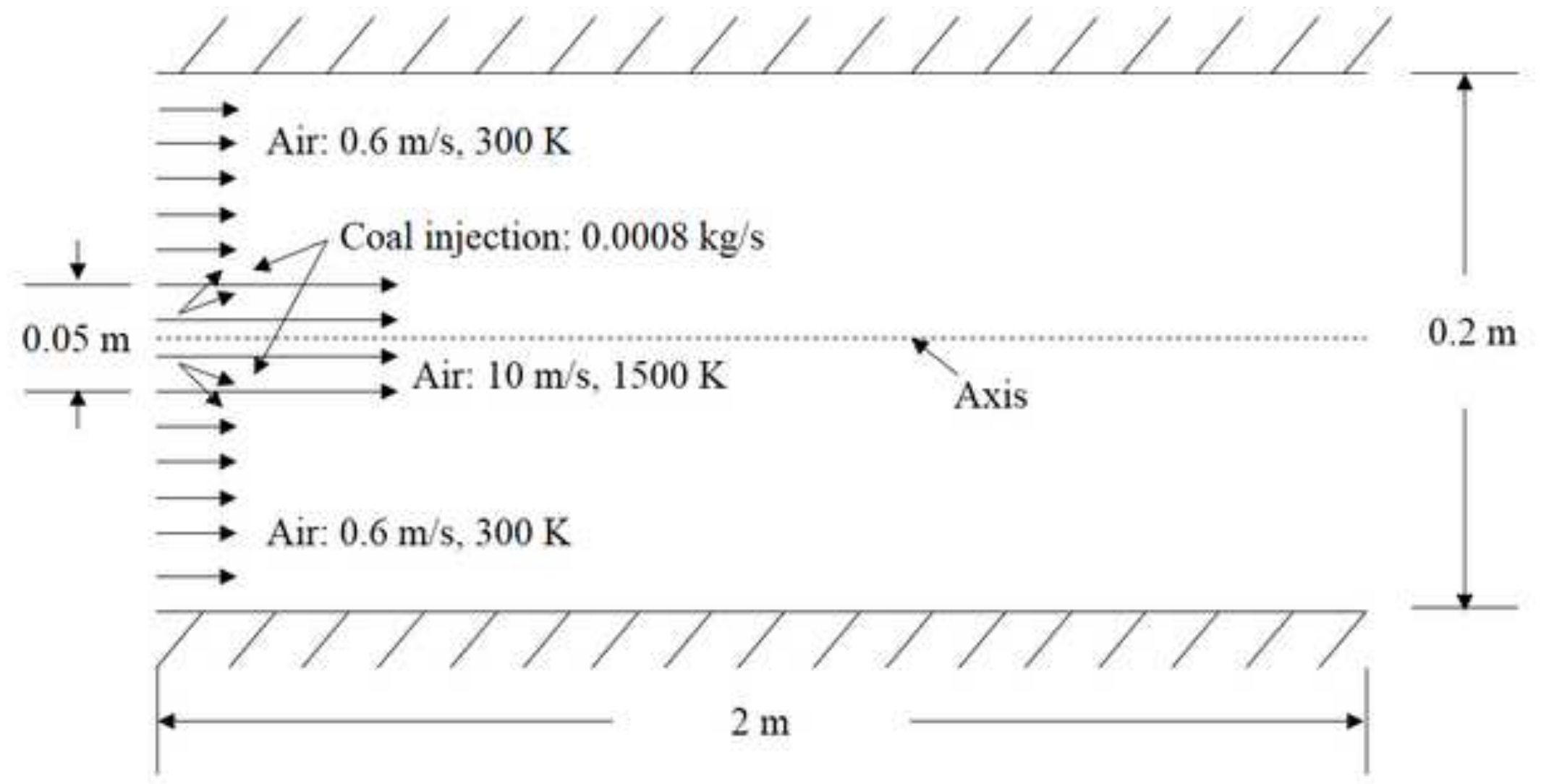




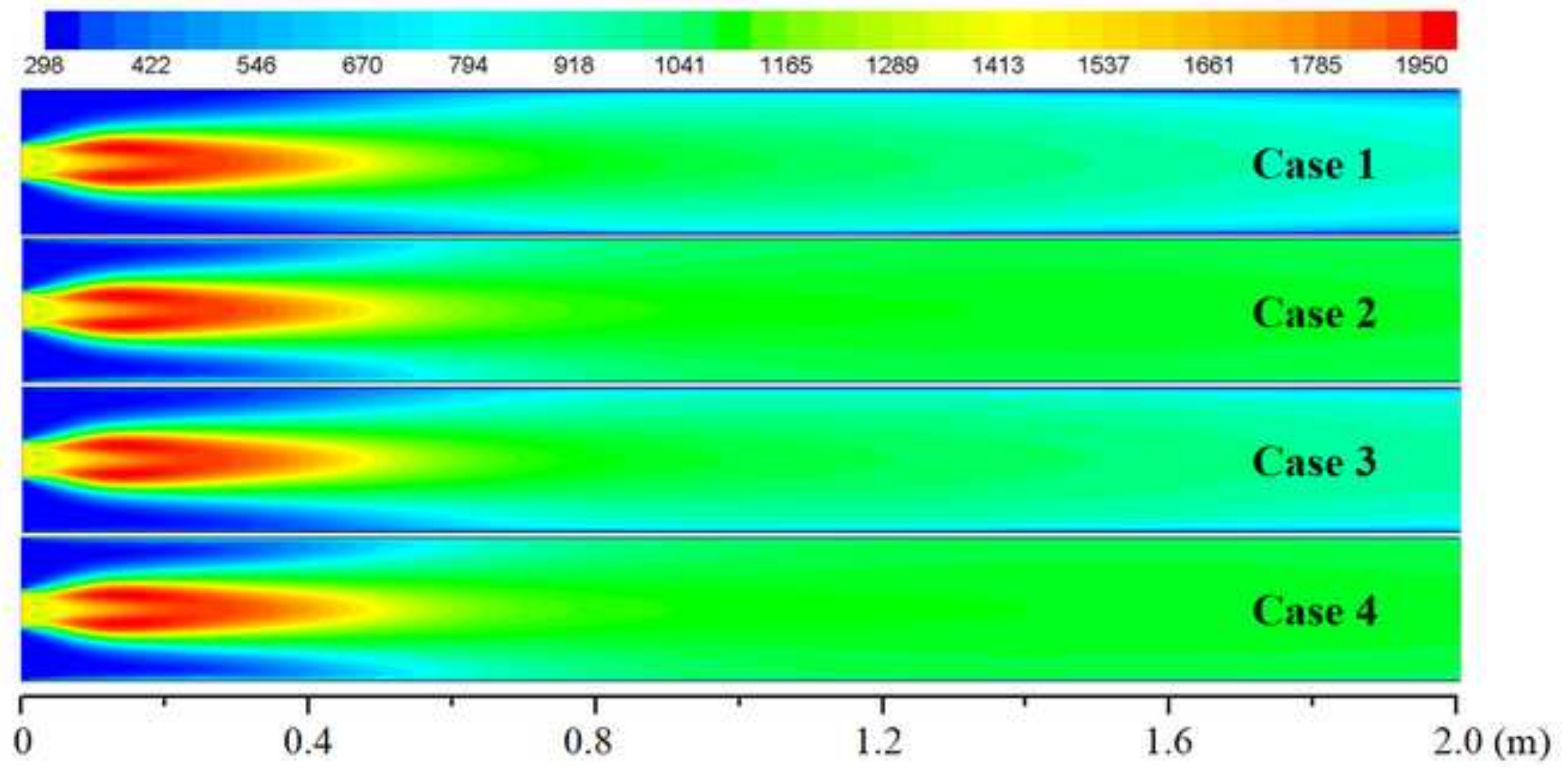



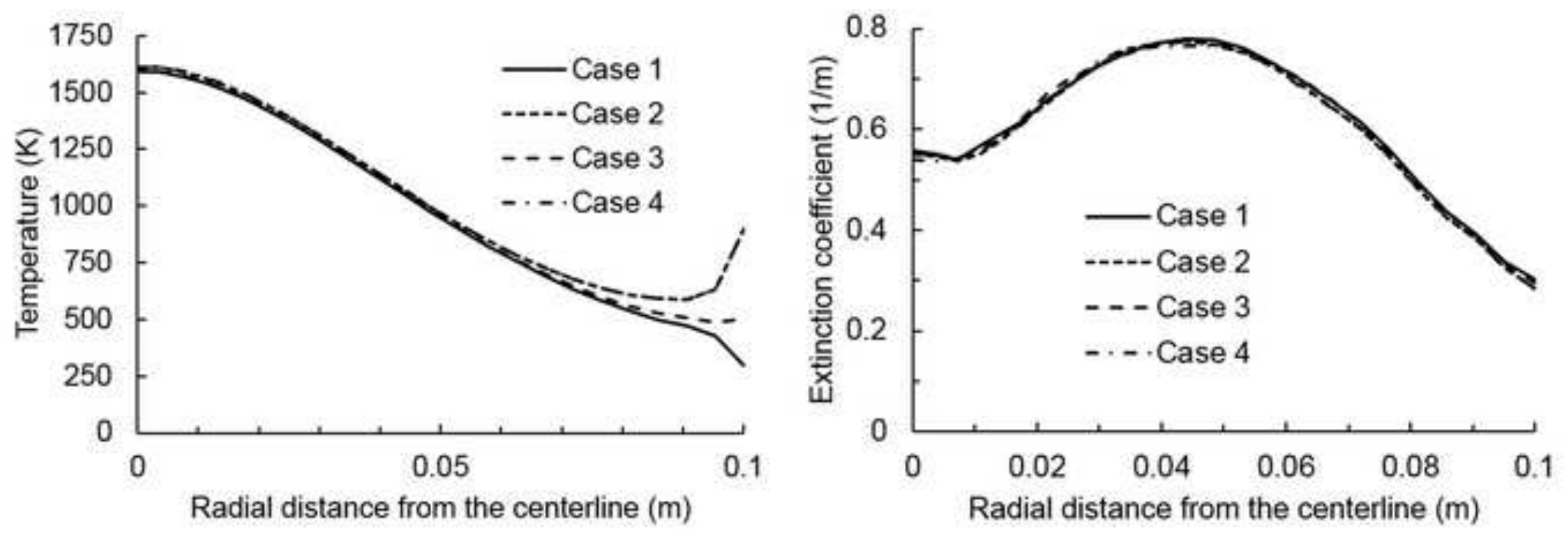


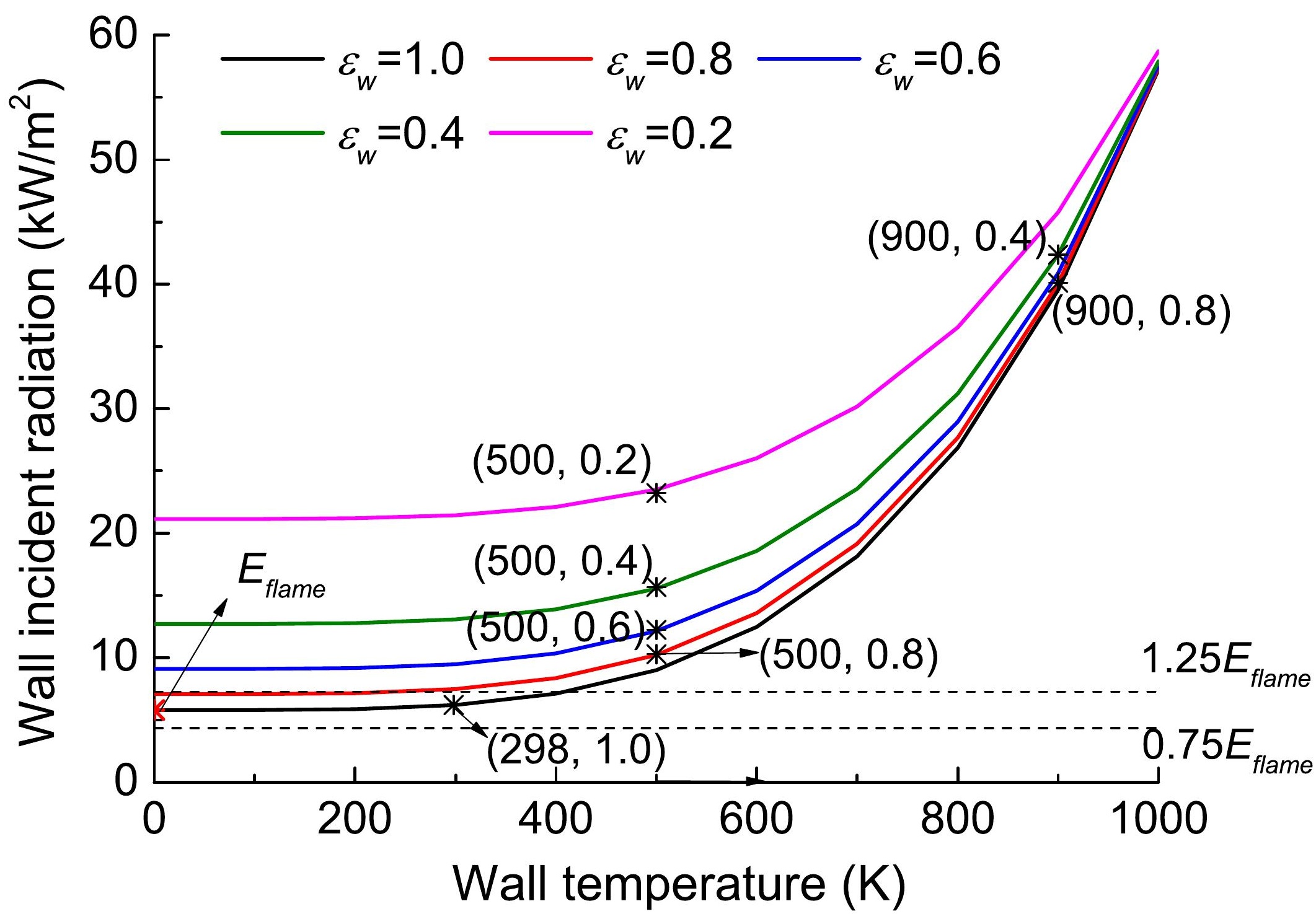




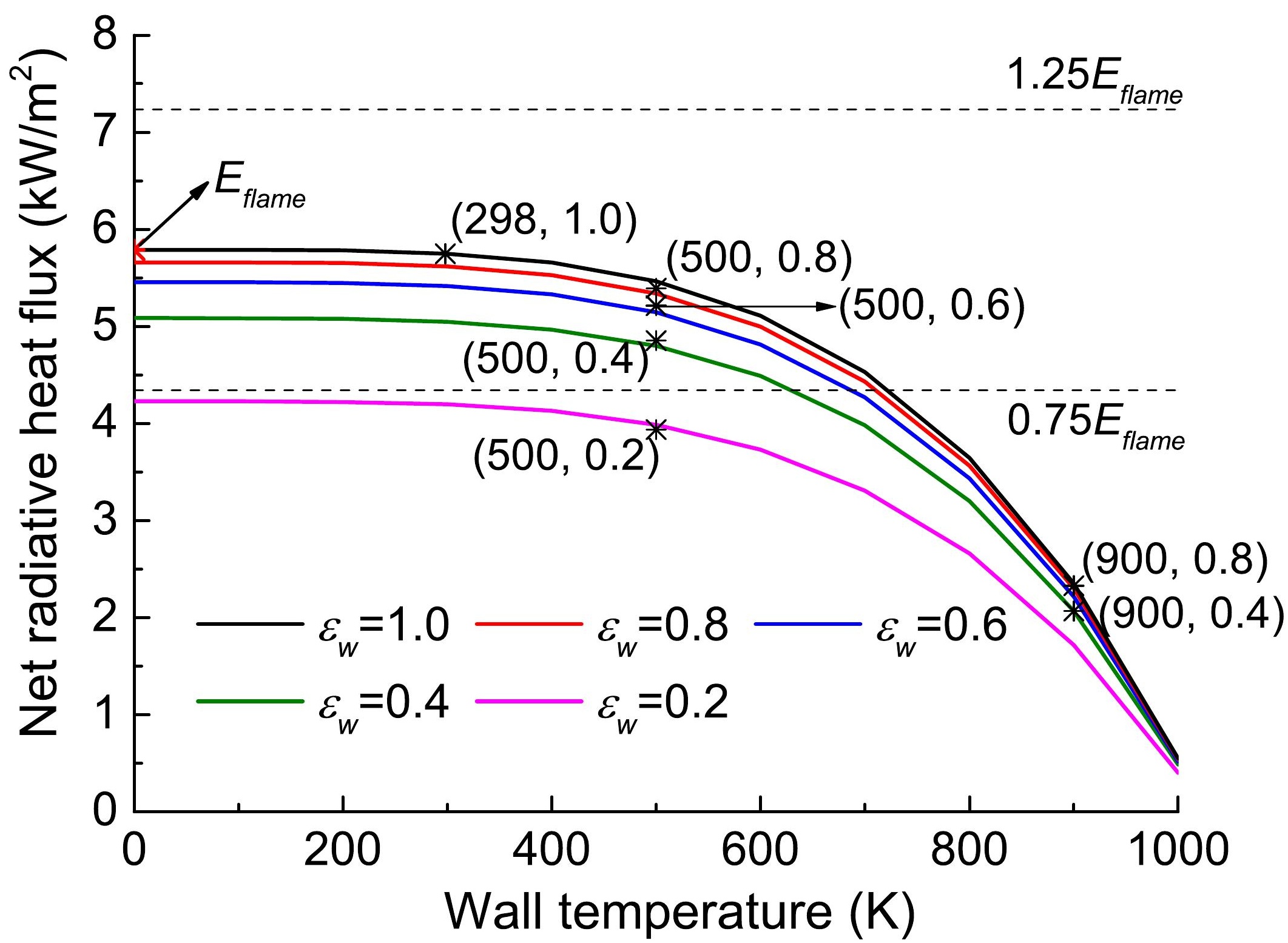




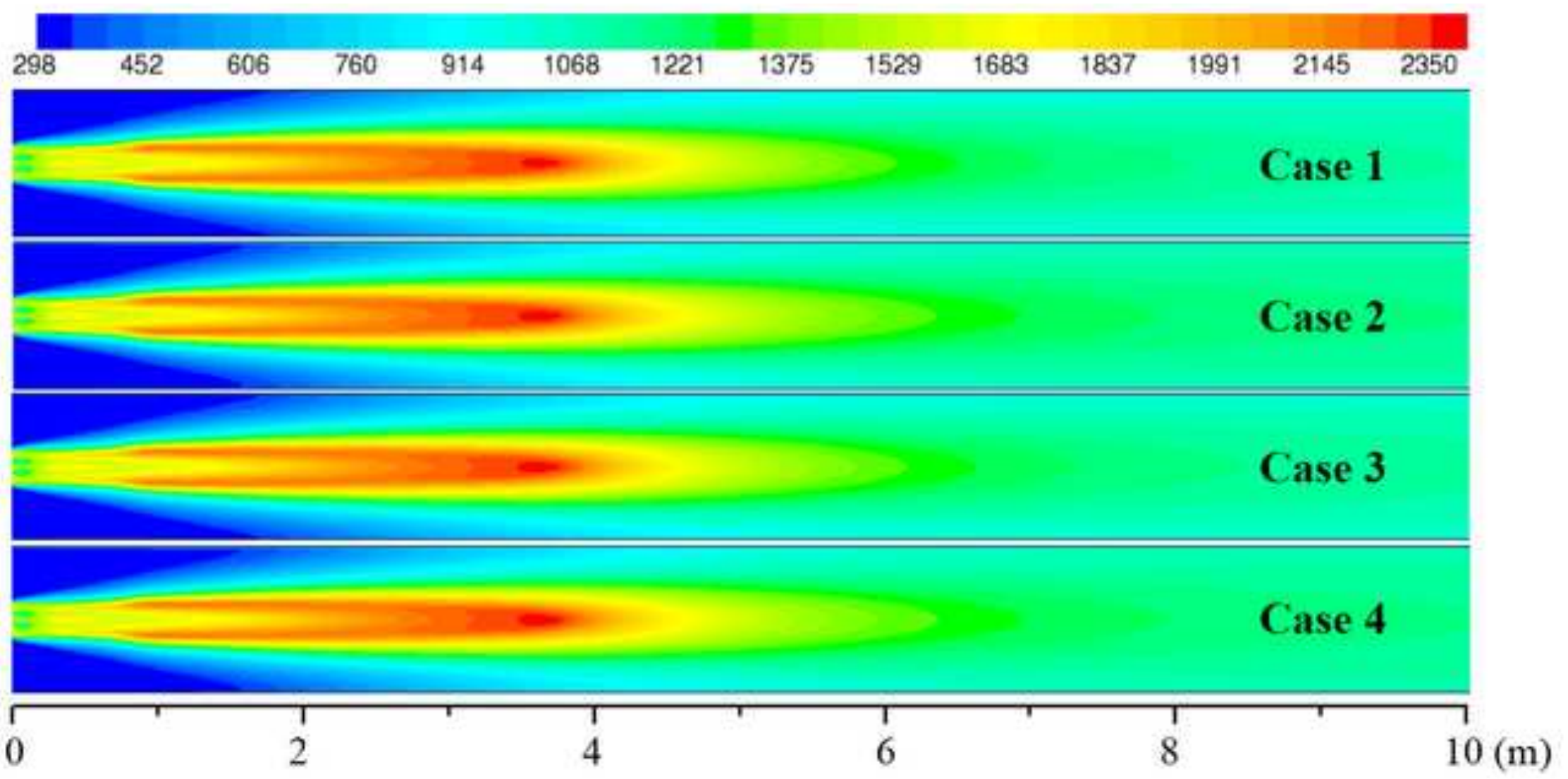



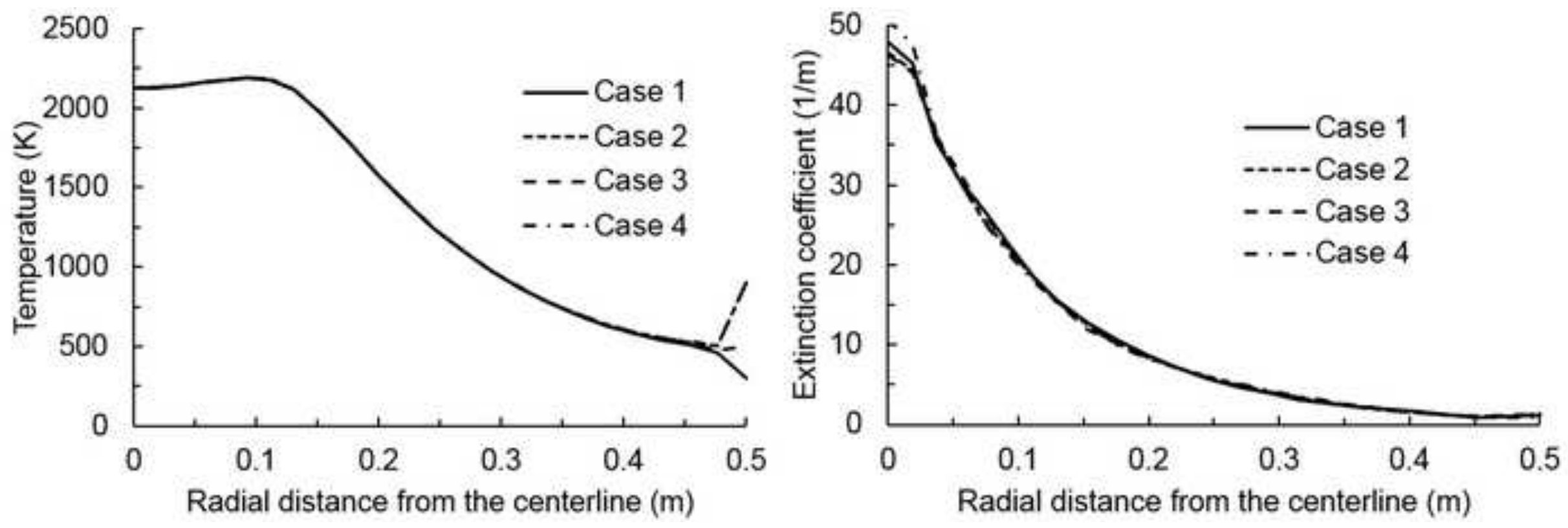


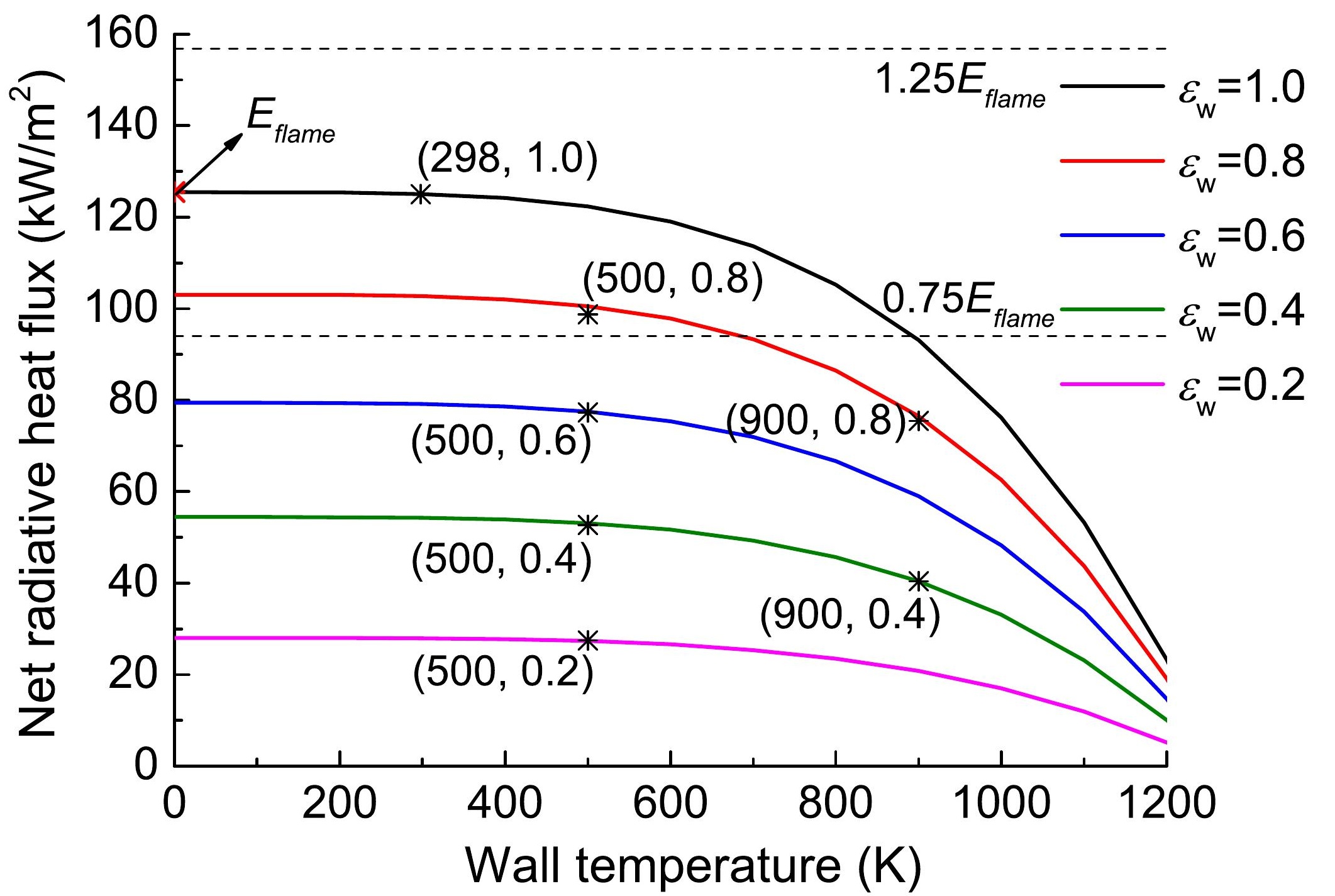


